\title{
Response Adaptation to Broadband Sounds in Primary Auditory Cortex of the Awake Ferret
}

\author{
Barak Shechter $^{a, *}$, Didier A. Depireux ${ }^{a, b}$ \\ ${ }^{a}$ Deptartment of Anatomy and Neurobiology, School of Medicine, University of \\ Maryland, Baltimore, 20 Penn St. HSF II Rm. S251, Baltimore, MD 21201 \\ ${ }^{\mathrm{b}}$ Program in Neuroscience, School of Medicine, University of Maryland, \\ Baltimore, Baltimore, MD 21201
}

\begin{abstract}
Driven by previous reports of adaptation to persistent stimuli in other brain regions, we investigated adaptive effects in the Primary Auditory Cortex of awake nonbehaving ferrets (Mustela Putorius furo). Electrophysiological data was obtained in response to the presentation of auditory gratings with a structured spectrotemporal envelope of varying bandwidth which had repeated transitions between low and high modulation depths. The responses were analyzed in terms of the evoked spike rates and in terms of the degree of phase locking to the modulation. We found two populations of cells, both of which showed adaptation in the traditional sense. For one population, we also found a second order of adaptation-i.e., adaptation of the adaptation. This suggests the existence of at least two coding strategies which differ in the weight placed on sensory context.
\end{abstract}

* Corresponding author.
Email address: bshec001@umaryland.edu (Barak Shechter). 


\section{Introduction}

The sensory environment is highly variable and gives rise to a broad spectrum of possible percepts. For a sensory system to provide useful information about the environment, it must be able to faithfully and reliably encode biologically relevant stimuli. However, owing to the comparatively narrow range of neural outputs, an efficient system must employ a strategy between two extreme possibilities: static or adaptive coding. Static coding implies having multiple functional units, each responding to a narrow range of the stimulus spectrum; when taken together, they span all possible stimulus values. By contrast, an adaptively coding unit shifts its input/output function based on local stimulus statistics, but always has a narrower range of outputs in comparison to the full stimulus spectrum (Reinagel, 2001; Smirnakis et al., 1997; Dean et al., 2005). While the latter is a more efficient way of representing the sensory environment, it is only possible when stimulus statistics are constrained to a narrow enough range over the timescale of neural coding (Laughlin, 1981; de Ruyter van Steveninck et al., 1994; DeWeese and Bialek, 1995). In practice, both coding strategies are found concurrently. For example, distinct retinal ganglion cells encode visual information arriving from distinct locations, and each cell encodes the mean light intensity adaptively (Shapley and EnrothCugell, 1984).

It has been shown that adaptation of neural output functions is not restricted to occur only in response to changes in the mean value of the input. For instance, neurons in the early visual pathway also adapt in response to changes in the variance (contrast) of input signals (Smirnakis et al., 1997; Kim and Rieke, 2001), and neurons in the auditory pathway adapt to changes in the kur- 
tosis (Kvale and Schreiner, 2004). Adaptation has been shown to occur along different stations of the visual pathway, from the retina (Baccus and Meister, 2002) to primary visual cortex (Crowder et al., 2006) and higher (Kohn and Movshon, 2003).

Adaptation is also highly dependent on the stimulus context. The adaptation seen to a particular stimulus can change as a function of the ensemble of stimuli it is presented in (Ulanovsky et al., 2004; Werner-Reiss et al., 2006; Bartlett and Wang, 2005). Furthermore, these different adaptations occur on different time scales. Just as the degree of adaptation is dependent on both the stimulus and its context, so too does the time scale of adaptation depend on both the stimulus and its context. Adaptation to new stimulus conditions occurs on a time scale comparable to the optimal detector (Fairhall et al., 2001)

In the auditory pathway, the vast majority of adaptation studies have concentrated on responses at the level of the auditory nerve with few investigations of higher processing centers along the auditory pathway: it is now well established that auditory nerve fibers exhibit adaptive responses when presented with pure tone pips (Rhode and Smith, 1985). Higher up, and driven by phenomena described in the visual pathway, Kvale and Schreiner recently conducted a study of the Inferior Colliculus (IC) looking for responses which adapted to changes in the higher order statistics of auditory stimuli (Kvale and Schreiner, 2004). When presented with a modulated tone, IC neurons adapted their response according to the statistics of the modulation envelope (e.g. mean, variance, kurtosis). However, little is known regarding adaptation in auditory cortex, and even less is known about the effects of varying bandwidths on adaptive coding. 
Adaptation has also been shown psychophysically in the auditory modality. In (Wojtczak and Viemeister, 2003), for instance, human subjects were asked to match perceived equivalent amplitude modulation depths of two pure tones, before and after prolonged exposure to one of the tones at full modulation. The perceived modulation depth was reduced after exposure to the adaptor that was modulated at the same rate as the standard. The difference in judgement between pre- and post-exposure was most marked when the modulation depth of the two tones was about $60 \%$ (the lowest modulation measured) but there was little effect for larger modulation depths of the probe tones or after exposure to a fully-modulated tone with a different frequency of modulation.

Primary auditory cortex is generally thought to encode properties of the spectral envelope of sound (Shamma et al., 1993; Kowalski et al., 1996a; Eggermont, 2001). Cells in the different auditory cortical fields have been shown to respond to modulations of different carrier waveforms independent of the specific carrier chosen (Schreiner and Urbas, 1986, 1988), even though there is a differential response with respect to the type of carrier used (Eggermont, 1994). The modulations found in speech (Chi et al., 1999), music (Klein et al., 2003) and natural sounds (Attias and Schreiner, 1997) are exactly the same range of modulations to which Primary Auditory Cortex responds best. Further developing the response properties to modulations, these everyday complex sounds can be thought of as linear superpositions of dynamic auditory gratings (Kowalski et al., 1996a,b), also known as ripples. Knowing the neural responses to a basic set of auditory gratings, one can characterize the response properties of a cell (Depireux et al., 2001; Klein et al., 2000) to complex sounds around a given level.

Cortical neurons encode envelope modulations in two schemes simultaneously: 
- temporal locking to the envelope and - spike rate modulation (Eggermont, 1994; Bieser and Muller-Preuss, 1996; Liang et al., 2002). However, how these neurons modify their use of these two coding schemes over time to adapt to their sensory environment is still unclear. In this paper, we studied how primary auditory cortex neurons adapt to a sudden change in the modulation depth of tones, narrow- and broad-band stimuli, both in their overall firing rates and the locking to the modulations. We also show that during prolonged presentations of alternating modulation depths (low-high and high-low), we found adaptation to the adaptation; in other words, the amount of adaptation of a given neuron for a specific transition typically becomes smaller as the number of transitions increases.

\section{Methods}

\subsection{Surgical Procedures}

Surgery was performed under halothane anesthesia on six 3-12 month old ferrets (Mustela putorius furo) of either sex. 10 cc of Ringer's solution was injected subcutaneously both at the start and at the end of the procedure to help metabolic stability. Heart rate, blood oxygen saturation and body temperature were monitored throughout. The scalp was resected and both temporalis muscles were removed. Craniotomies were performed over the left and right auditory cortices. To prevent neogenesis of the dura, the mitotic inhibitor 5-flurouracil (5-FU) was applied (Spinks et al., 2003). Three animals were chronically implanted with multi-electrode arrays loaded each with up to eight independently adjustable electrodes (Neuralynx, Inc., Tuscon, AZ), and 
in three animals, the craniotomies were enclosed with sterile wells filled with sterile aCSF. Screws were put in the skull to help anchor dental cement to the skull. The experimental apparatus and headbars were fixed in place with additional dental cement. 5-FU was applied daily for the well preparation. The Neuralynx electrode holder system was sealed. All experimental procedures were approved by the University of Maryland Animal Care and Use Committee and were in accord with NIH Guidelines on the case and use of laboratory animals.

\subsection{Electrophysiology}

Neurophysiological recordings were conducted in awake, usually restrained, non-behaving animals. Parylene-coated tungsten electrodes with polyimide tubing (Micro Probe, Inc., Gaithersburg, MD, 3.0M $\Omega-5.0 M \Omega$ at $1 k H z$ ) were used in both the electrode array and well preparations. Data were collected with a Cheetah Data Acquisition System (Neuralynx, Inc., Tuscon, AZ). The signal was bandpass filtered with low and high cutoff frequencies of $300 \mathrm{~Hz}$ and $6 \mathrm{kHz}$, respectively. Data were stored for offline spike sorting using the software program MClust (Redish, 2004) coupled with purpose designed Matlab software. The actual quantitative analysis of the data is described below. Recording sessions lasted between 2 and 4 hours. Treats were given to the animals between stimulus presentations or as deemed needed from a closed-circuit camera in order to maintain a state of wakefulness. 


\subsection{Stimulus Set}

Cells were initially characterized by their pure tone response. A randomized series of $150 \mathrm{~ms}$ pure tone pips was played ( 5 octaves, base frequency $200 \mathrm{~Hz}, 30-$ 60 dB SPL). Then, Spectro-Temporal Response Fields (STRF) were measured with Temporally Orthogonal Ripple Combinations (TORC) stimuli, as previously described by (Depireux et al., 2001; Klein et al., 2000). Once cells were characterized, the stimuli used to study adaptation consisted of moving auditory gratings, also called ripples, varying in bandwidth, modulation frequency and modulation depth; briefly, a grating (see Fig. 1) has a spectral profile consisting in a sinusoidal modulation around a mean level on a $\log$ (frequency) axis, and can be characterized by its spectral density $(\Omega)$ in cycles/octave, drift velocity $(w)$ in $\mathrm{Rad} / \mathrm{sec}$ (referred to as $\mathrm{Hz}$ in the following) and modulation depth $(\Delta A)$. Specifically, each stimulus consisted of repeating components of a given density, velocity and bandwidth, with alternating low and high modulation depths. The grating was centered on the tonotopic axis around the best frequency of the neuron, and was built from $1 \mathrm{~Hz}$ spaced carrier tones. The phase of each carrier tone was randomized, and power was rolled down by 3 $\mathrm{dB}$ per octave to keep a constant power per octave. The modulation of each carrier is shown in the spectrotemporal envelope of the stimulus (Fig. 1).

The amplitude $S(x, t)$ of each tone of frequency $f$, with $x=\log _{2}\left(f / f_{0}\right), f_{0}$ the lower edge of the spectrum, is then adjusted as

$$
S(x, t)=L[1+\Delta A \cdot \cos (2 \pi(\Omega \cdot x+w \cdot t))]
$$

for a linear modulation. $L$ is the overall intensity base of the stimulus and is adjusted to a level typically $10-15 \mathrm{~dB}$ above the lower threshold of the 
cell as determined with pure tones at the tonal best frequency. Note that when both $\Omega$ and $w$ are positive, the corresponding grating envelope travels towards the low frequencies, and that since the tones that make up a grating are logarithmically spaced, its pitch is indeterminate.

[ Fig 1 around here ]

\subsection{Sound Presentation}

Sound waveforms were generated in Matlab and synthesized on a Tucker Davis Technologies RX6 DSP processor (Tucker Davis, Alachua, Florida). Stimuli were presented free-field in a double-walled sound attenuation chamber (Industrial Acounstics, New York, with a noise isolation class of 70dB) with a Manger transducer fixed directly above the the animal's head (Manger, Mellrichstadt, Germany) at a fixed sampling rate of $100 \mathrm{kHz}$.

\subsection{Data Analysis}

Peri-stimulus time histograms (PSTHs) of the neural reponse to the gratings were created by binning the data with 8 evenly spaced bins for every period of the grating modulation. 8-point Fourier transforms of the histograms were then computed for each period of the modulation. The neural response was first characterized by the following indices computed from the transform:

1. The transform $D C$, or average response, normalized. The $D C$ values computed for each modulation period within a stimulus were treated as a group and were scaled and shifted with respect to their mean $\mu$ and 
standard deviation $\sigma$. This is a measure of the magnitude (number of spikes) of the neural output responding to the stimulus in units of $\sigma_{D C}$. The normalization permits quantification of the adaptation for each cell in terms of $\sigma_{D C}$ of its firing rate distribution. This allows cells with different firing rates can be compared directly. (Eq. 2)

$$
\overline{D C}=\frac{D C-\mu_{D C}}{\sigma_{D C}}
$$

\section{The power in the first component of the Fourier transform} $\left(A C_{1}\right)$, or fundamental, normalized by the total transform power.

This is a measure of the fraction of the neural output responding in a locked fashion to the modulation of the stimulus. (Eq. 3)

$$
\overline{A C}_{1}=\frac{A C_{1}}{\sqrt{\sum_{i=1}^{4}\left|A C_{i}\right|^{2}}}
$$

where $A C_{i}$ is the magnitude of the $i^{\text {th }}$ component of the Fourier transform of a component. Note that for an uncorrelated response, the power is expected to be equally distributed across all its Fourier transform components $A C_{i}$. With the normalization of Eq. 3, the sum of the $\overline{A C}_{i}$ 's is 1 . Since we use the first four components of the response, the expected value of $\overline{A C}_{1}$ is $1 / 4$ for a random response. We also estimated the variance of each $\overline{A C}_{1}$ using a bootstrap method (Klein et al., 2006) with a seed of 50 repetitions, to use as a weight for the linear fits described below.

To analyze the change in adaptation over time, we computed linear fits of the response to each component of the stimulus. The high modulation depth components were separated from the low modulation depth components, and their respective fits were concatenated to form a continuous curve. This was done for both $\overline{D C}$ and $\overline{A C}_{1}$. The fits for $\overline{A C}_{1}$ were weighted by the bootstrap 
estimate of the variance of each point.

Since our hypothesis was that the rate of adaptation changes upon repetitive changes in modulation depth (Fig. 1), we fit the concatenation of component fits with a combination of a linear fit and an exponential fit. The linear fit, of the form $\mathbf{a} x+\mathbf{b}$, accounts for the possibility that no change in adaptation occurs from component to component, such that all component fits are identical in their slope and the $\mathbf{a} x+\mathbf{b}$ fit is the same for duration of the stimulus; the exponential fit, of the form $\mathbf{c}(\exp (\mathbf{d} x)-1)$, accounts for the possibility that the rate of adaptation for a given component is a function of the number of transitions that preceeded it.

Hence, the concatenations of a given modulation depth were best fit by a curve with both a linear and an exponential component:

$$
y=\mathbf{a} x+\mathbf{b}+\mathbf{c}\left(e^{\mathbf{d} x}-1\right)
$$

For most cells, the change in parameters $\overline{D C}$ and $\overline{A C}_{1}$ was well fit by the above function. In Fig. 2, we show the analysis for a single cell. The PSTH of the cell is shown in the first row; note that in this extreme example, the low modulation is actually a zero percent modulation, i.e. the stimulus consists in unmodulated noise during the low-modulations. Also, for reasons of clarity, we chose to display an example with only 6 components. Most cells were presented with a stimulus containing 12 components. The results for such an analysis are shown in Fig. 3, where we concatenated component fits obtained in a similar manner to that shown in Fig. 2.

We compared the distributions of parameters $\mathbf{a}, \mathbf{c}$, and $\mathbf{d}$ computed for the responses to stimuli of different bandwidths and different modulation depths. 
b was not considered essential to the analysis, it was used as an overall correction factor for the constraints inherent in Eq. 4. In particular, because of our construction, the concatenations formed from Eq. 2 and Eq. 3 always start at zero.

\section{Results}

\subsection{Single Unit Responses to Individual Stimulus Components}

Peri-stimulus time histograms were constructed at time bins corresponding to $1 / 8$ of the grating periodicity. The measures described above were then computed from this data by Fourier analysis. The $\overline{D C}$ of the response to each component of the stimulus always decayed. We fit the $\overline{D C}$ with both an exponential decay function and a linear function, and found that the linear fit was best able to capture the decay of the response (Fig. 2D). During periods of high modulation, the magnitude of $\overline{A C}_{1}$ was usually close to 1 , suggesting that most of the response power was following the modulation function. In other words, the response was phase locked to the spectro-temporal envelope modulations. This was also observed in the phase of $\overline{A C}_{1}$. In general terms, the phase of the response to high modulation gratings was well constrained to a narrow range with minor fluctuations. In contrast, during periods of low modulation depth, both the magnitude and phase of $\overline{A C}_{1}$ were seen to vary more randomly. (Fig. 2A-C). Points for which $\left|\overline{A C}_{1}\right| \leq 0.25$ were rejected because they were attributed to chance.

[ Fig 2 around here ] 


\subsection{Second Order Adaptation - DC}

To examine the change over time in the adaptation - which we call second order adaptation - of the overall spiking rate, we concatenated linear fits of the $\overline{D C}$ computed from the high and low modulation component responses. We then found the parameters which best fit Eq. 4 to the concatenation. The distribution of parameters $\mathbf{a}, \mathbf{c}$, and $\mathbf{d}$ were analyzed in reference to the modulation depth and bandwidth of the evoking stimulus.

[ Fig 3 around here ]

\subsubsection{Bandwidth}

In order to explore the contributions of Center Frequency $(\mathrm{CF})$ region versus the surround, usually inhibitory non-CF region, we decided to explore the effect of bandwidth on the second order adaptation. We therefore compared responses to stimuli of different bandwidth by grouping data points into one of three categories: $B W=0,0<B W \leq 2$, and $B W>2$ (octaves). Two dimensional distributions (low modulation vs. high modulation) corresponding to the three categories were compared using a Kolmogorov-Smirnov (KS) test. For parameters $\mathbf{a}$ and $\mathbf{c}$, the distributions were not significantly different across bandwidths $(P>0.30$ and $P>0.54$ respectively). Parameter $\mathbf{d}$ distributions for $B W=0$ and $0<B W \leq 2$ were significantly different $(P<0.01)$ with this test, but neither was significantly different than the distribution for $B W>2$ $(P>0.14)$ (Fig. 4).

[ Fig 4 around here ] 


\subsubsection{Low vs. High Modulation}

We then considered the effect of modulation depth on the fit parameter distributions, disregarding the three bandwidth categories which we concluded to be equivalent for this purpose (see previous section). Parameter a was biased towards negative values in both high and low modulations. In periods of low modulation, a had a mean of $-0.11 \pm 0.35 \sigma_{D C} s^{-1}$, and shifted toward more negative values with high modulation depths $\left(-0.20 \pm 0.31 \sigma_{D C} s^{-1}\right)$ (Fig. 5).

[Fig 5 around here]

Inspection showed the presence of two populations of responses with respect to parameters $\mathbf{c}$ and $\mathbf{d}$, this latter parameter characterizing the decay constant of the exponential (see Fig 5, right column). The first population was characterized by $|\mathbf{d}| \leq 0.006 \mathrm{~s}^{-1}$ (that is, 1/d larger than about 160 seconds, i.e. non-adapting), for which $\exp (\mathbf{d} x)-1 \simeq \mathbf{d} x$, which is itself small; furthemore, Eq. 4 is reduced to the form $(\mathbf{a}+\mathbf{c} \mathbf{d}) x+\mathbf{b}$ so that the relative values of $\mathbf{a}, \mathbf{c}$ and $\mathbf{d}$ cannot be determined. $\mathbf{c}$ therefore became substantially large in absolute value, ranging from -200 to $200 \sigma_{D C}$ in both high and low modulation. In this population of responses, there was little to no change in the spike rate adaptation with repeated presentation of the stimulus components (Fig. 8), i.e. there was no second-order adaptation.

In the remainder of the responses, the distribution of parameter $\mathbf{c}$ ranged from -5 to $5 \sigma_{D C}$ and was biased towards positive values. Upon transition from low to high modulation depth, there was a slight overall negative shift in the distribution (Fig. 5, Fig. 6, Fig. 7).

[Fig 6 around here] 
[Fig 7 around here]

[Fig 8 around here]

The distributions in $\mathbf{d}$ were asymmetrical and ranged from -3 to $.2 s^{-1}$. There were very few data points for which $\mathbf{d}>0.006 \mathrm{~s}^{-1}$, and so, excluding the population of linear responses (since we already addressed the points for which $|\mathbf{d}| \leq 0.006 s^{-1}$ ) we next analyzed $\mathbf{d}$ between -3 and $-0.006 s^{-1}$. Since we expect second order adaptation to reduce the in-component adaptation as a function of component number, $\mathbf{d}$ is expected to have a biased negative distribution, so that the distributions were fit to a curve of the form:

$$
\alpha_{\mathbf{d}}\left(x-x_{0}\right) e^{\beta_{\mathbf{d}}\left(x-x_{0}\right)}
$$

For low modulations, $\mathbf{d}$ had a broader negative tail, whereas for high modulations, the distribution fell off more sharply. This was seen as an increase in both parameters $\alpha_{\mathbf{d}}$ and $\beta_{\mathbf{d}}$ (Fig. 5).

All parameter space distributions were significantly different between periods of high and low modulation depths (KS, $P<0.001)$.

\subsection{Second Order Adaptation - $A C_{1}$}

To examine the change over time in phase locking to the stimulus, we concatenated linear fits of the measure of locking, $\overline{A C}_{1}$, in a similar manner as was done for the $D C$. As before, we found the parameters which best fit Eq. 4 to the concatenation, and their distributions were analyzed with respect to the different stimulus conditions. 


\subsubsection{Bandwidth}

Data points were grouped into the same three bandwidth categories used when analyzing the $D C$ of the response: $B W=0,0<B W \leq 2$, and $B W>2$ (octaves). The resultant two dimensional distributions (low modulation vs. high modulation) were compared using a Multivariate Analysis of Variance (MANOVA) test. None of the distributions were significantly changed by varying bandwidth $(P>0.51[\mathbf{a}], P>0.20[\mathbf{c}]$, and $P>0.37[\mathbf{d}])$ (Fig. 9).

[Fig 9 around here]

\subsubsection{Low vs. High Modulation}

Since bandwidth was an irrelevant factor in adaptation of phase locking we compared parameter distributions with respect only to transitions in modulation depth. The parameter a distribution had a mean of $0 \pm 0.06 s^{-1}$ for low modulation depths, and shifted towards more negative values upon transition to high modulation depths $\left(-0.01 \pm 0.06 s^{-1}\right)$ (Fig. 10).

[Fig 10 around here]

As with the $D C$, two populations of responses were found with respect to parameters $\mathbf{c}$ and $\mathbf{d}$ : non-adapting and adapting. The first population was characterized by $|\mathbf{d}| \leq 0.015 \mathrm{~s}^{-1}$ (for a $1 / \mathbf{d}$ value of about 60 seconds). As before, we note that $\exp (\mathbf{d} x)-1 \simeq \mathbf{d} x$ in this regime, so that the corresponding c values ranged from -100 to 50 in both high and low modulation depths because the fitting problem was poorly defined in this regime. The combination of small $\mathbf{d}$ and large $\mathbf{c}$ reduced the exponential part of Eq. 4 to be approximately linear. Cells displaying these responses would be expected to show no 
change in their adaptation of phase locking to the stimulus components with each transition between modulation depths. (Fig. 13).

[Figs 11, 12 and 13 around here]

The distribution of parameter $\mathbf{c}$ ranged from -1 to 1 for the adapting responses. As in the case of the $D C$, there was an overall negative shift in the distribution of $\mathbf{c}$ values with the transition from low to high modulation depth. (Fig. 10, Fig. 11, Fig. 12).

As before, the distributions in $\mathbf{d}$ were asymmetrical and ranged from -3 to $.2 s^{-1}$. We compared the distributions for adapting responses and found a slight positive shift in $\mathbf{d}$ values, but the shape of the distribution remained relatively unchanged upon either transition from low to high modulation depth or from high to low modulation depth. (Fig. 10).

The distributions of $\mathbf{a}$ and $\mathbf{c}$ were significantly different for periods of high and low modulation depths (KS, $P<0.05)$. In contrast to the results obtained for the $D C$ of the response, distribution for $\mathbf{d}$ did not significantly change with the transitions in modulation depth (KS, $P>0.26)$.

\section{Discussion}

The initial objective in this study was to find cortical correlates to the reports of adaptation found along the auditory pathway. While we found correlates of adaptation (decay of firing rate) on a cell by cell basis, and found that over the 2 seconds of each component we did get short term adaptation of the neural response, we did not find a unique pattern of adaptation over these compo- 
nents, (see Fig 2), nor a predominant rate of decay for most cells, unlike (Kvale and Schreiner, 2004) who found a reliable decay constant of $300 \mathrm{~ms}$ in the inferior colliculus, albeit for a difference type of stimulus. We found no reliable asymetry when comparing low-to-high versus high-to-low transitions either (data not shown). Since it is generally thought that auditory cortex encodes modulations of the spectro-temporal envelope of incoming sounds (Schreiner and Urbas, 1986, 1988; Eggermont, 1994; Shamma et al., 1993; Kowalski et al., 1996a,b), we decided to look at the higher orders of adaptation to repeated presentations of alternating low and high modulations - an adaptation to adaptation.

\subsection{Exponental Fitting vs. Linear Fitting}

Spike rate adaptation is generally thought to follow an exponential decay time course; however, this is largely an empirical result and not based upon a single unit or network model. When examining the first order of adaptation in AI to the components, we found that the decay in spike rate was described equally well by a linear fit or an exponential decay function (measured by the mean square distance between the fits and the data). There are two main reasons to which this can be attributed:

1. Adaptation time scale. Many studies have shown a wide range of time scales for spike rate adaptation, varying from tens of milliseconds to minutes (Fairhall et al., 2001) (Ulanovsky et al., 2004). In the event that the first order adaptation occured on a time scale longer than any of the stimulus components, we would expect the response not to asymptote to a steady state value by the end of the component. This initial part of the 
response would be fit reasonably well by a straight line since the higher order terms of the expansion of $e^{-\frac{x}{\tau}}$ would be dominated by the lower order terms.

$$
e^{-\frac{x}{\tau}}=\sum_{n=0}^{\infty}(-1)^{n} \frac{x^{n}}{\tau^{n} \cdot n !}=1-\frac{x}{\tau}+\frac{x^{2}}{2 \tau^{2}}-\frac{x^{3}}{6 \tau^{3}} \cdots
$$

2. Noise Error. Steady-state cortical response are quite variable upon repeated presentation of the same stimulus, producing a level of "noise" in the resulting measurements. If the level of noise in the response is comparable to the order of magnitude of the adaptation, the asymptotic time course will be obscured relative to the initial decay. However, a linear fit will still reveal the overall trend strength of the underlying adaptation.

Since we wanted to study the change in the adaptation to repeated transitions in modulation depth, we only needed to characterize the overall trend of the first order adaptation, and therefore the linear fit to the adaptation of single components was quite sufficient.

\subsection{Rebounding Responses vs. Decaying Responses}

In previous adaptation studies of the auditory and visual systems, the majority of adapting responses either had large positive transients followed by a decay to steady state or large negative transients followed by a rebound to steady state. Which type of response was seen depended on the particular transition used to perturb the neuron (Smirnakis et al., 1997; Fairhall et al., 2001; Kvale and Schreiner, 2004). However, units which respond to perturbation only with a large positive transient and consequent decay to steady state have also been observed (Smirnakis et al., 1997). In our experiments, neurons showed no 
rebounding responses to any transition. The majority of adapting responses found in this study almost exclusively decayed in both high to low and low to high transitions. This suggests perhaps that the function of the cortical network at $\mathrm{AI}$ is to detect a change in modulation, but not the direction of change.

\subsection{Adapting vs. Non-Adapting Responses}

Two clear populations of responses were found in parameters $\mathbf{c}$ and $\mathbf{d}$. The two were split based on a critical value of $\mathbf{d}$. For values of $\mathbf{d}$ corresponding to a very long second order adaptation $(1 / \mathbf{d}>160 \mathrm{sec}$, compared to the 20 seconds typical of the total duration of our stimulus), the combination of $\mathbf{c}$ and d parameters reduced the exponential part of Eq. 4 to a linear function. These units did not use stimulus context as part of their input/output functions.

The distinction must be made between non-adapting in the local sense, versus non-adapting in the broad sense. The units characterized here as non-adapting (broad) did adapt on a per-component basis. However, these units did not change their response to these components with each successive presentation. Non-adapting here refers to the lack of second order adaptation. From the point of view of a model of AI, it is the existence of units with no second order adaptation that matters, even though they were not prevalent.

\subsection{Excitatory Responses}

There is an inherent bias in the selection procedure for candidate recordings which favors primarily excitatory cells (since we searched for cells using a 
random pure tone stimulus). Based on half peak width vs. half trough width ratios, we believe that all units recorded from were excitatory cells. Bruno and Simons (2002) studied the correlation of neural spike waveform parameters with the excitatory or inhibitory properties of the cells. Our spike waveforms had wide initial peaks $(>300 \mu s e c)$ and troughs $(>400 \mu s e c)$. Following Bruno and Simons conclusions, we only recorded from excitatory cells.

\subsection{Multimodal Parameter Distribution for c}

When looking closely at the distribution in $\mathbf{c}$ for the $D C$ of the response, we were able to distinguish multiple populations of values, each population being fit by a Gaussian envelope. For low modulation depths, the parameter distribution was best fit by a sum of three Gaussian envelopes, whereas for high modulation depths, the best fit was achieved with two envelopes. We were unable to find any correlation between these populations or with the other parameters. The significance of these multi-modal distributions is unknown. It was not seen for the $A C_{1}$ of the response.

\subsection{Contextual Importance}

The model presented here suggests the role of cells in primary auditory cortex in detection of novel contrast changes in the auditory environment. As a sound is repeatedly encountered, the transition to that sound becomes less and less novel, and thus, the adaptation to that particular sound changes with each presentation. Perhaps a related effect was seen by Ulanovsky et. al. where they report a stimulus specific adaptation (Ulanovsky et al., 2004). They found that 
the degree of adaptation to a particular component of the stimulus depended on its context and statistics in the whole stimulus. A drawback present in the analysis is the removal of the transient response. In order to analyze the second order adaptation, we needed to remove absolute spike rates, and look only at relative rates (this was achieved by concatenating the linear fits). Thus, there is an ambiguity in the instantaneous rate change evoked by a transition, but the overall change is captured.

\section{Acknowledgment}

This research was supported by NIH grant RO1 DC-05937. We also wish wish to thank Yadong Ji for help with surgery and recordings, Peter Marvit, Heather Dobbins, Sridhar Kalluri for help with the experiments and Asaf Keller for help with the manuscript.

\section{References}

Attias, H., Schreiner, C. E., 1997. Low-order temporal statistics of natural sounds. In: Advances in Neural Information Processing Systems. pp. 27-33.

Baccus, S. A., Meister, M., 2002. Fast and slow contrast adaptation in retinal circuitry. Neuron 36 (5), 909-919.

Bartlett, E. L., Wang, X., 2005. Long-lasting modulation by stimulus context in primate auditory cortex. J.Neurophys. 94 (3), 83-104.

Bieser, A., Muller-Preuss, P., 1996. Auditory responsive cortex in the squirrel monkey: neural responses to amplitude-modulated sounds. Exp.Brain Res. $108(2), 273-84$. 
Bruno, R. M., Simons, D. J., Dec 2002. Feedforward mechanisms of excitatory and inhibitory cortical receptive fields. J Neurosci 22 (24), 10966-75.

Chi, T., Gao, Y., Guyton, M. C., Ru, P., Shamma, S., 1999. Spectro-temporal modulation transfer functions and speech intelligibility. J.Acoust.Soc.Am. $106(5), 2719-32$.

Crowder, N. A., Price, N. S., Hietanen, M. A., Dreher, B., Clifford, C. W., Ibbotson, M. R., 2006. Relationship between contrast adaptation and orientation tuning in v1 and v2 of cat visual cortex. J.Neurophys. 95, 271-83. de Ruyter van Steveninck, R. R., Bialek, W., Potters, M., Carlson, R. H., 1994. Statistical adaptation and optimal estimation in movement computation by the blowfly visual system. Proc. IEEE Int. Conf. Systems, Man, and Cybernetics, 302-7.

Dean, I., Harper, N. S., McAlpine, D., 2005. Neural population coding of sound level adapts to stimulus statistics. Nat Neurosci. 8 (12), 1684-9.

Depireux, D. A., Simon, J. Z., Klein, D. J., Shamma, S. A., 2001. Spectrotemporal response field characterization with dynamic ripples in ferret primary auditory cortex. J.Neurophys. 85 (3), 1220-34.

DeWeese, M., Bialek, W., 1995. Information flow in sensory neurons. Il Nuovo Cimento 17D, 733-41.

Eggermont, J. J., 1994. Temporal modulation transfer functions for am and fm stimuli in cat auditory cortex. effects of carrier type, modulating waveform and intensity. Hear.Res. 74 (1-2), 51-66.

Eggermont, J. J., 2001. Between sound and perception: reviewing the search for a neural code. Hear.Res. 157 (1-2), 1-42.

Fairhall, A. L., Lewen, G. D., Bialek, W., de Ruyter Van Steveninck, R. R., 2001. Efficiency and ambiguity in an adaptive neural code. Nature 412 (6849), 787-92. 
Kim, K. J., Rieke, F., Jan 2001. Temporal contrast adaptation in the input and output signals of salamander retinal ganglion cells. J.Neurosci. 21 (1), 287-99.

Klein, D. J., Depireux, D. A., Simon, J. Z., Shamma, S. A., 2000. Robust spectrotemporal reverse correlation for the auditory system: optimizing stimulus design. J.Computat.Neurosci. 9 (1), 85-111.

Klein, D. J., Koenig, P., Koerding, K. P., 2003. Sparse spectrotemporal coding of sounds. EURASIP J.Applied Signal Processing 7, 659-67.

Klein, D. J., Simon, J. Z., Depireux, D. A., Shamma, S. A., 2006. Stimulusinvariant processing and spectrotemporal reverse correlation in primary auditory cortex. J.Computat.Neurosc., accepted.

Kohn, A., Movshon, J. A., 2003. Neuronal adaptation to visual motion in area mt of the macaque. Neuron 39 (4), 681-91.

Kowalski, N., Depireux, D. A., Shamma, S. A., 1996a. Analysis of dynamic spectra in ferret primary auditory cortex. i. characteristics of single-unit responses to moving ripple spectra. Journal of Neurophysiology. 76 (5), 350323.

Kowalski, N., Depireux, D. A., Shamma, S. A., 1996b. Analysis of dynamic spectra in ferret primary auditory cortex. ii. prediction of unit responses to arbitrary dynamic spectra. J.Neurophys. 76 (5), 3524-34.

Kvale, M. N., Schreiner, C. E., 2004. Short-term adaptation of auditory receptive fields to dynamic stimuli. Journal of Neurophysiology 91 (2), 604-12.

Laughlin, S., Sep-Oct 1981. A simple coding procedure enhances a neuron's information capacity. Z.Naturforsch.[C] 36 (9-10), 910-2.

Liang, L., Lu, T., Wang, X., May 2002. Neural representations of sinusoidal amplitude and frequency modulations in the primary auditory cortex of awake primates. J.Neurophysiol. 87 (5), 2237-61. 
Redish, A. D., 2004. Mclust: a spike-sorting toolbox, freely available software. http://www.cbc.umn.edu/ redish/mclust.

Reinagel, P., 2001. Neurobiology: The many faces of adaptation. Nature 412 (6849), 776-7.

Rhode, W. S., Smith, P. H., May 1985. Characteristics of tone-pip response patterns in relationship to spontaneous rate in cat auditory nerve fibers. Hear.Res. 18 (2), 159-68.

Schreiner, C. E., Urbas, J. V., 1986. Representation of amplitude modulation in the auditory cortex of the cat. i. the anterior auditory field (aaf). Hear.Res. 21 (3), 227-41.

Schreiner, C. E., Urbas, J. V., 1988. Representation of amplitude modulation in the auditory cortex of the cat. ii. comparison between cortical fields representation of amplitude modulation in the auditory cortex of the cat. i. the anterior auditory field (aaf). Hear.Res. 32 (1), 49-63.

Shamma, S. A., Fleshman, J. W., Wiser, P. R., Versnel, H., 1993. Organization of response areas in ferret primary auditory cortex. J.Neurophys. 69 (2), $367-83$.

Shapley, R., Enroth-Cugell, C., 1984. Visual adaptation and retinal gain controls. Progress in Retinal Research 3, 263-346.

Smirnakis, S. M., Berry, M. J., Warland, D. K., Bialek, W., Meister, M., 1997. Adaptation of retinal processing to image contrast and spatial scale. Nature 386 (6620), 69-73.

Spinks, R. L., Baker, S. N., Jackson, A., Khaw, P. T., Lemon, R. N., 2003. Problem of dural scarring in recording from awake, behaving monkeys: a solution using 5-fluorouracil. J.Neurophys. 90 (2), 1324-32.

Ulanovsky, N., Las, L., Farkas, D., Nelken, I., 2004. Multiple time scales of adaptation in auditory cortex neurons. J Neurosci 24 (46), 10440-53. 
Werner-Reiss, U., Porter, K. K., Underhill, A. M., Groh, J. M., 2006. Long lasting attenuation by prior sounds in auditory cortex of awake primates. Exp Brain Res 168 (1-2), 272-6.

Wojtczak, M., Viemeister, N. F., 2003. Suprathreshold effects of adaptation produced by amplitude modulation. J. Acoust. Soc. Am. 114 (2), 991-7. 


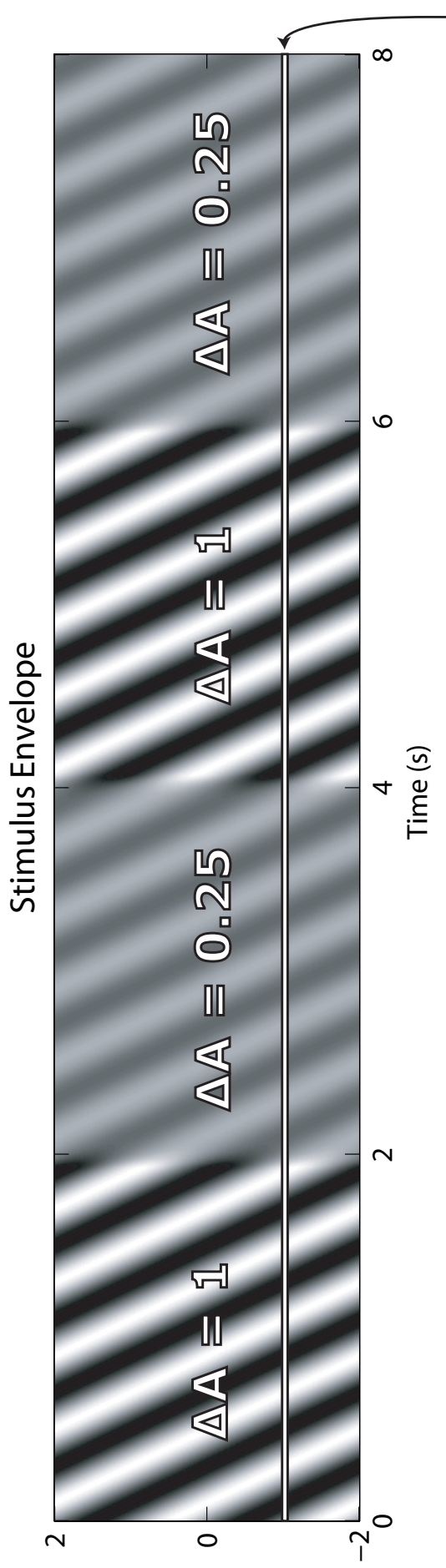

(รәлељวо) КЈиәnbән

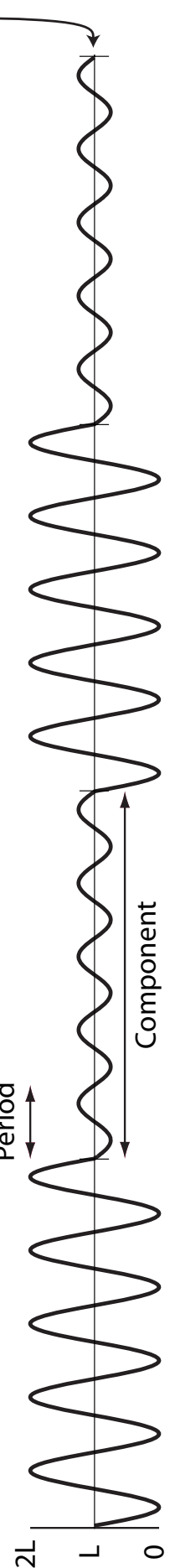

(gp) |әләך

Fig. 1. Spectro-temporal envelope of a typical stimulus. The stimulus shown has four components of two seconds each. The modulation depths of the components alternate between high $(100 \%$, indicated by $\Delta A=1)$ and low $(25 \%$, indicated by $\Delta A=0.25)$ values. This stimulus has a spectral density of 0.5 cycles/octave, a velocity of $2.5 \mathrm{~Hz}$, and a bandwidth of 4 octaves (frequencies are relative to the best frequency of the cell being stimulated). The cross section below shows the modulation envelope of the frequency component specified. Typical stimuli were of longer duration and therefore consisted in more components. 


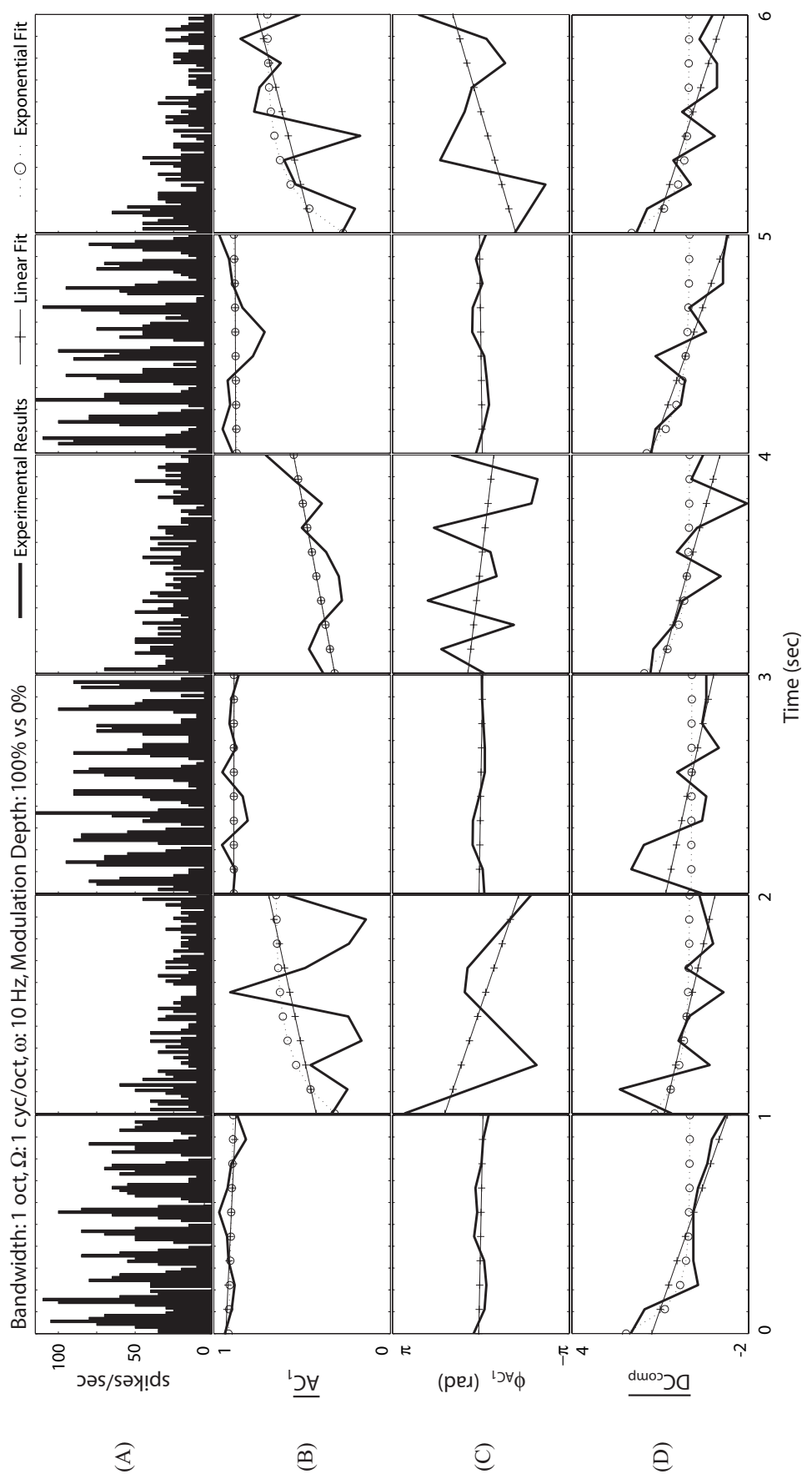

Fig. 2. Fourier Analysis of Single Unit Activity. A. Peri-stimulus time histograms of the data were constructed by binning the data every $1 / 8$ period of the temporal modulation (12.5 msec for this cell). B-C. Magnitude $\left(A C_{1}\right)$ and phase $\left(\Phi_{A C 1}\right)$ of the Fourier transform of each component. for each 8 bins of the PSTH in $(\mathbf{A})$. During high modulation depth components, the response is phase locked to the stimulus, which can be seen by $\overline{A C}_{1}$ having a magnitude of about 1 and being relatively constant in phase $\Phi_{A C 1}$. In contrast, during low modulation depth components, the response was more random with respect to stimulus timing. D. $D C$ for each 8 bins of the PSTH in (A). For the ease of comparison, the distributions of $D C$ for each component were normalized by subtracting the component mean and dividing by the component standard deviation $\left(D C-\mu_{\text {comp }}\right) / \sigma_{\text {comp }}$. The $D C$ decayed during each component regardless of modulation depth. 
Changes in Adaptation with Presentation Number
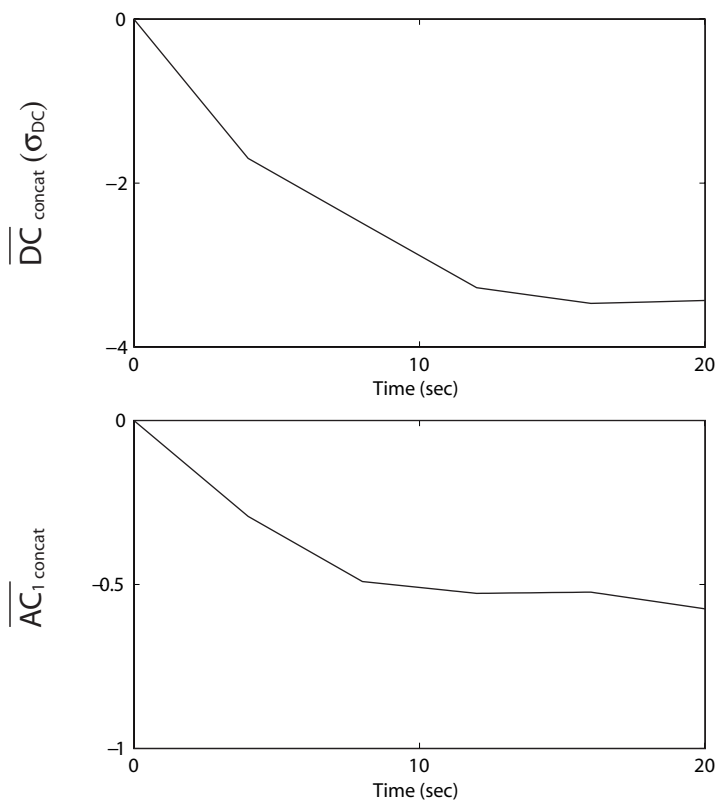

No Changes in Adaptation with Presentation Number
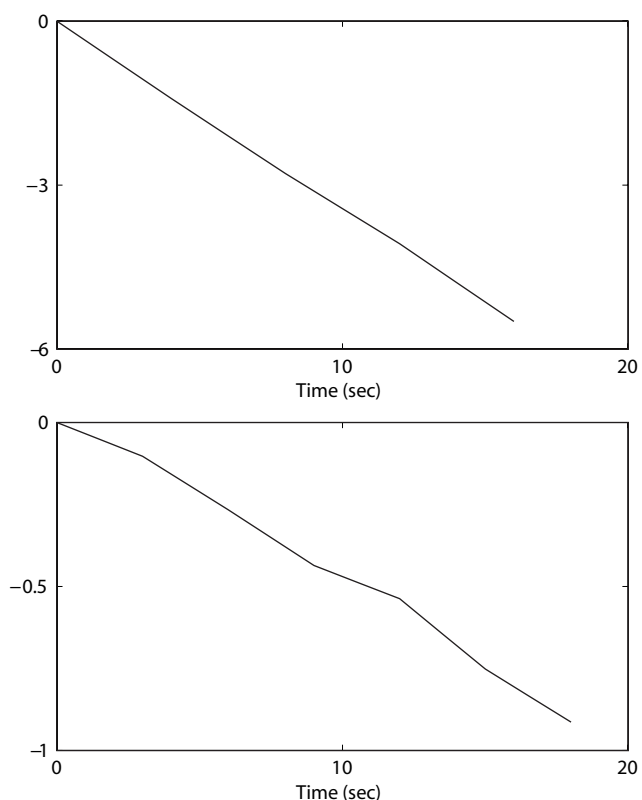

Fig. 3. Sample responses observed experimentally. In the top and bottom rows, the $D C$ and $A C_{1}$, respectively, were normalized and concatenated as described in the methods (Eqs. 2,3). On the left are sample responses with a second order of adaptation. The fit of the form of Eq. 4 will contain both linear and exponential parts. On the right are sample responses for which only first order adaptation was observed. In other words, there was no change in adaptation over time, and therefore, the fit contained only a linear part. 

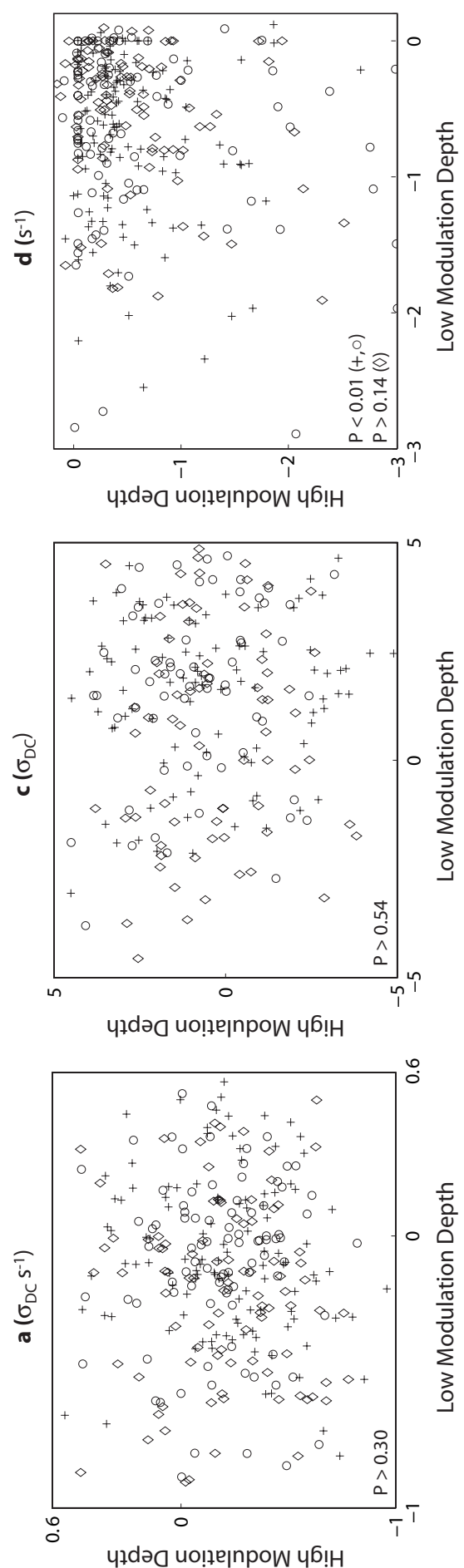

Fig. 4. Distributions of second-order adaptation parameter values for the $D C$ of the response grouped by stimulus bandwidth. The parameters for the low modulation depth components of the stimulus are plotted against the parameters for the high modulation depth components. The distributions for parameters a and $\mathbf{c}$ were found not to be significantly different with respect to the bandwidth of the stimulus (KS, $P>0.30$ and $P>0.54$, respectively). Distributions for $\mathbf{d}$ were found to be significantly different for $B W=0$ with respect to $0<B W \leq 2(P<0.01)$, but neither of these conditions differed significantly from $B W>2(P>0.14) . B W=0$ $(+), 0<B W \leq 2(\circ), B W>2(\diamond)$. Bandwidth in octaves. 

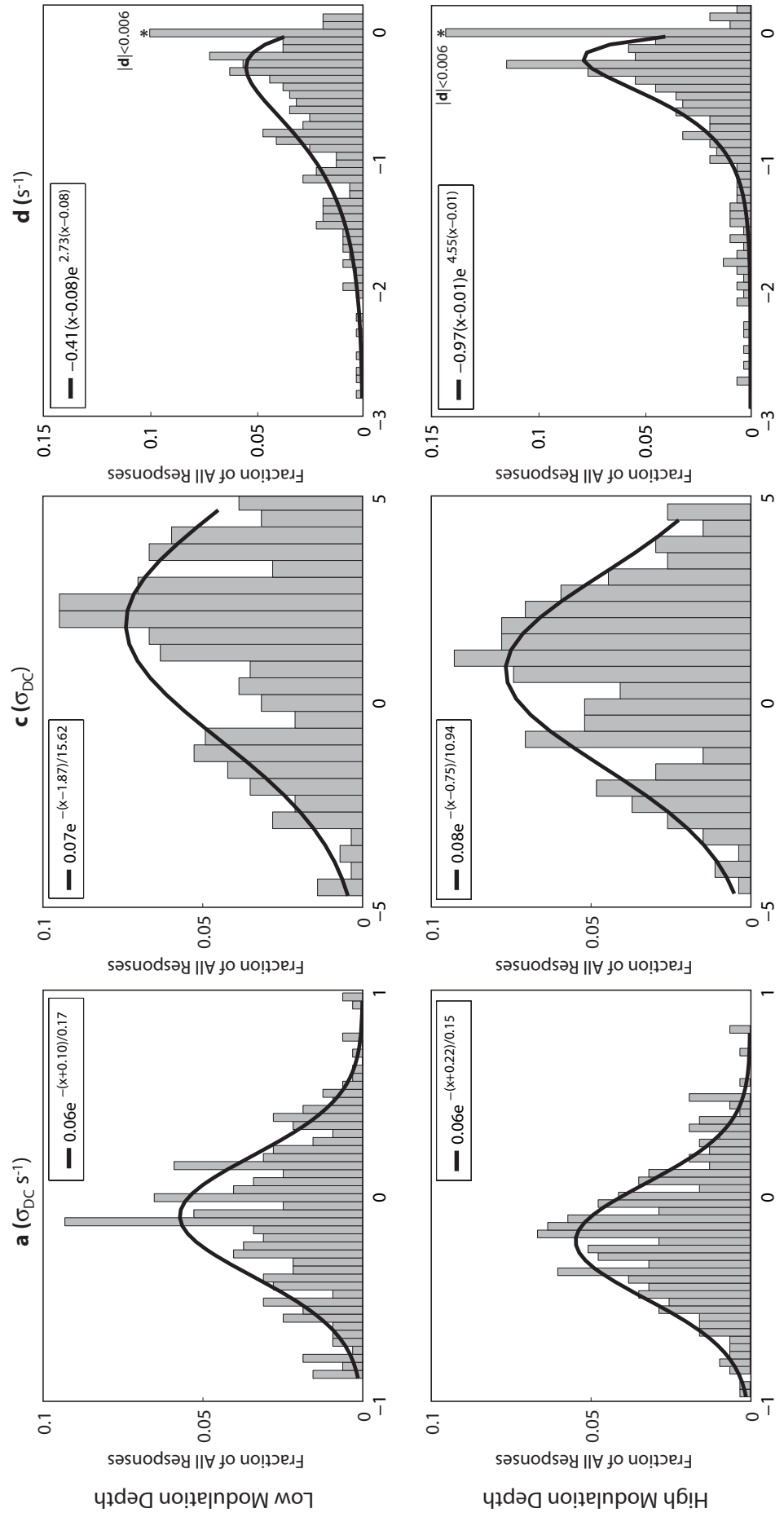

Fig. 5. Distributions of second-order adaptation parameter values for the $\overline{D C}$ of the response to stimuli with low and high modulation depths. For low modulation depths, the mean of a was $-0.11 \pm 0.35 \sigma_{D C} s^{-1}$, whereas for high modulation depths, it was $-0.20 \pm 0.31 \sigma_{D C} s^{-1}$. There were two populations observed in $\mathbf{c}$ and $\mathbf{d}$-nonadapting (for which $|\mathbf{d}|<0.006 \mathrm{~s}^{-1}$ ) and adapting (for which $|\mathbf{d}|>0.006 \mathrm{~s}^{-1}$ ). In the adapting responses, $\mathbf{c}$ had a mean of $1.04 \pm 2.29 \sigma_{D C}$ and $0.48 \pm 2.06 \sigma_{D C}$ for low and high modulation depths, respectively. $\mathbf{d}$ was characterized by a broader distribution for low modulation depths than for high modulation depths. 

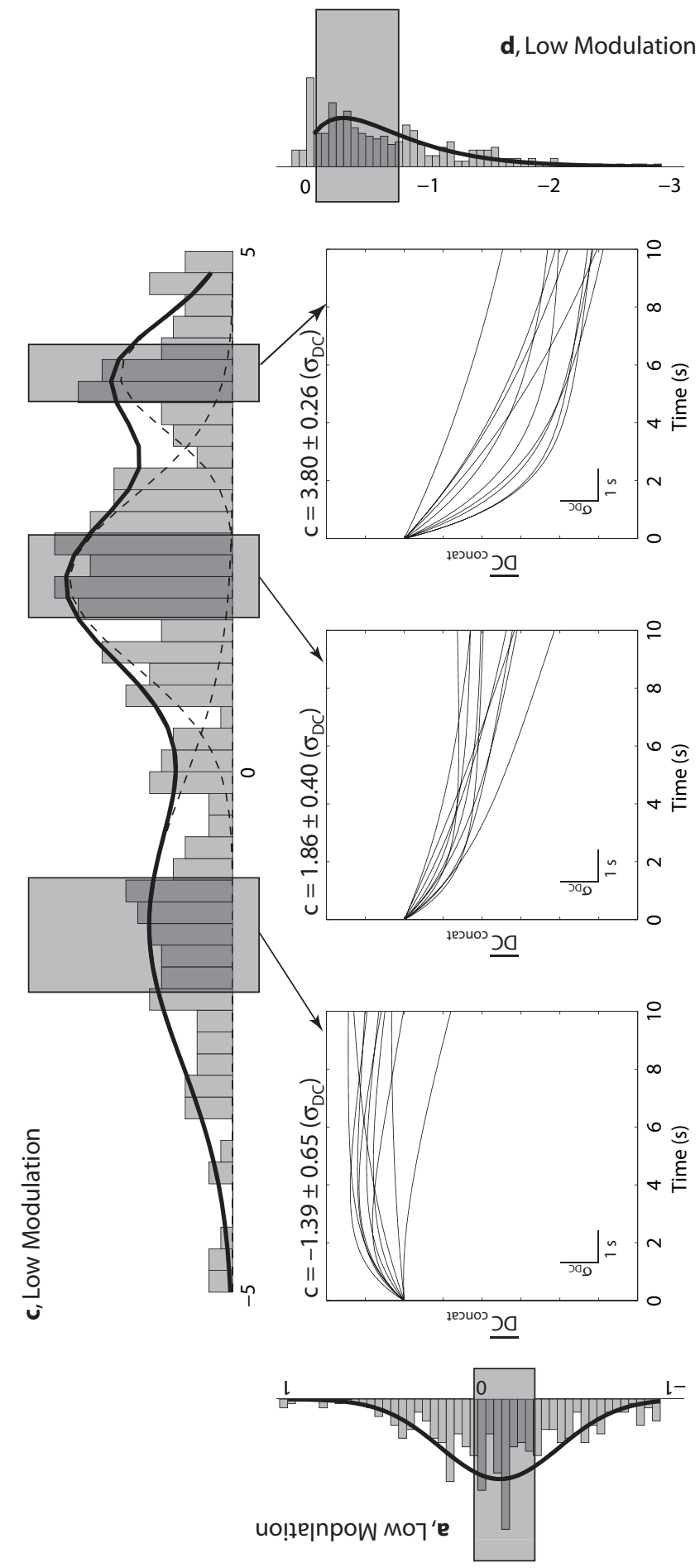

Fig. 6. Representative time-courses of the adaptation for the concatenated $\overline{D C}$ of the response to low modulation depth components computed from Eq. 4 with parameters $\mathbf{a}$, c, and $\mathbf{d}$, drawn from their respective distributions. When inspected closely, the distribution for $\mathbf{c}$ during periods of low modulation depth had three gaussian subpopulations. Sample groups were taken at the mean of each of these populations $\pm \sigma / 2$. Parameters $\mathbf{a}$ and $\mathbf{d}$ were selected in a similar manner. 


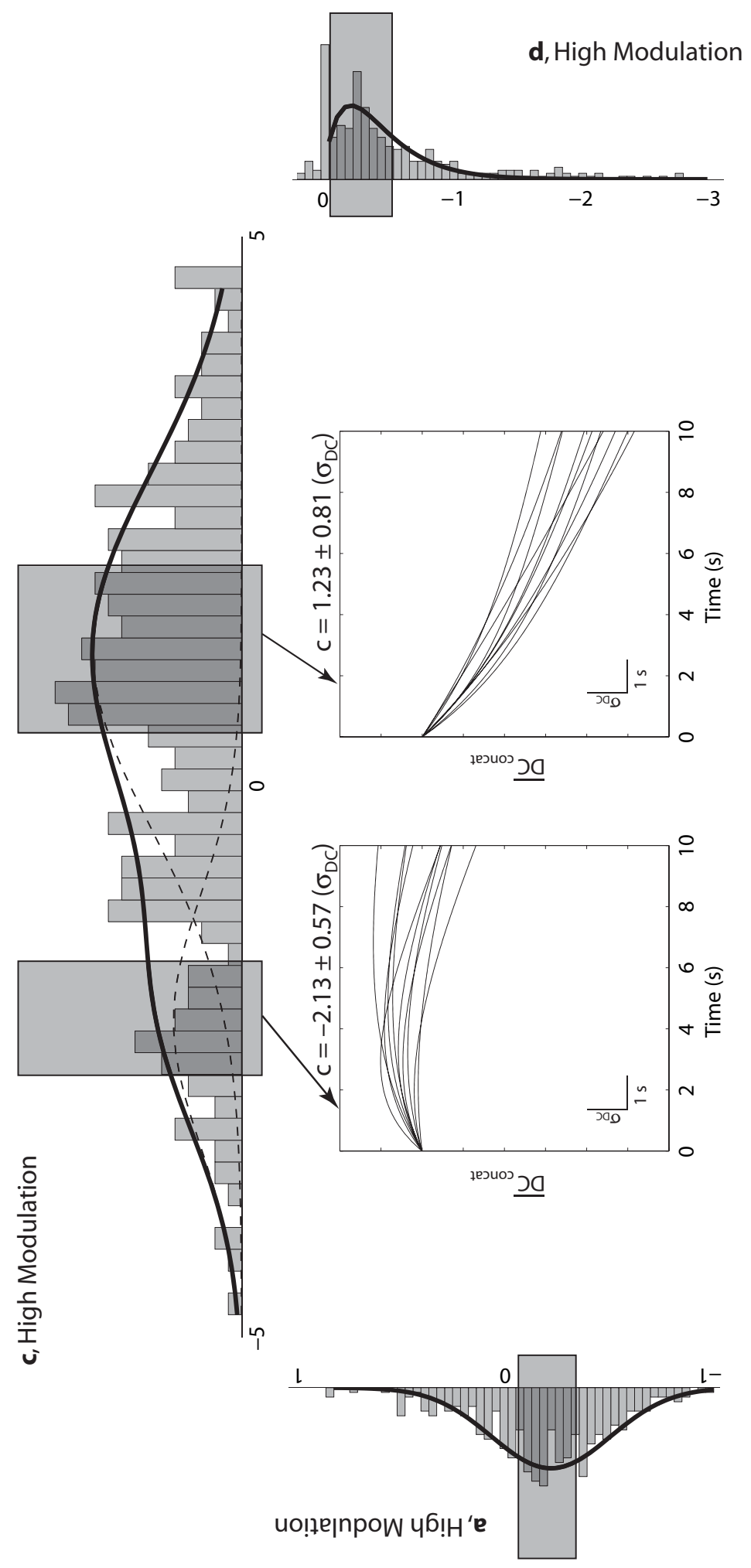

Fig. 7. Representative time-courses of the adaptation for the concatenated $\overline{D C}$ of the response to high modulation depth components computed from Eq. 4 with parameters a, c, and $\mathbf{d}$ drawn from their respective distributions. When inspected closely, the distribution for $\mathbf{c}$ during periods of high modulation depth had two gaussian subpopulations. Sample groups were taken at the mean of each of these populations $\pm \sigma / 2$. Parameters $\mathbf{a}$ and $\mathbf{d}$ were selected in a similar manner. 
$|d|<0.006 \mathrm{~s}^{-1}$

Low Modulation Depth

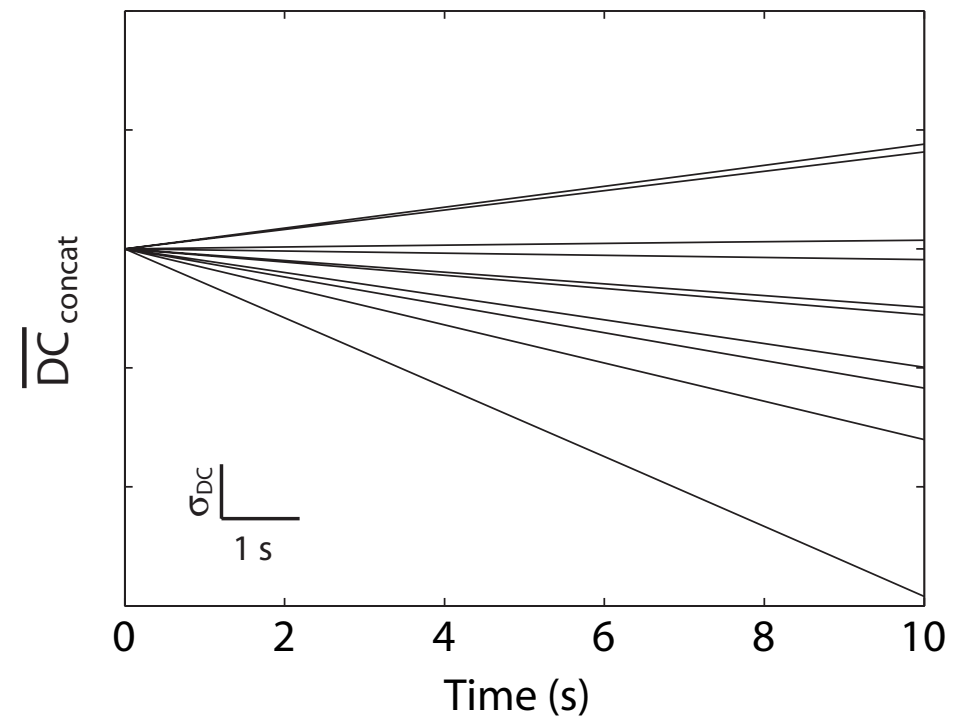

\section{High Modulation Depth}

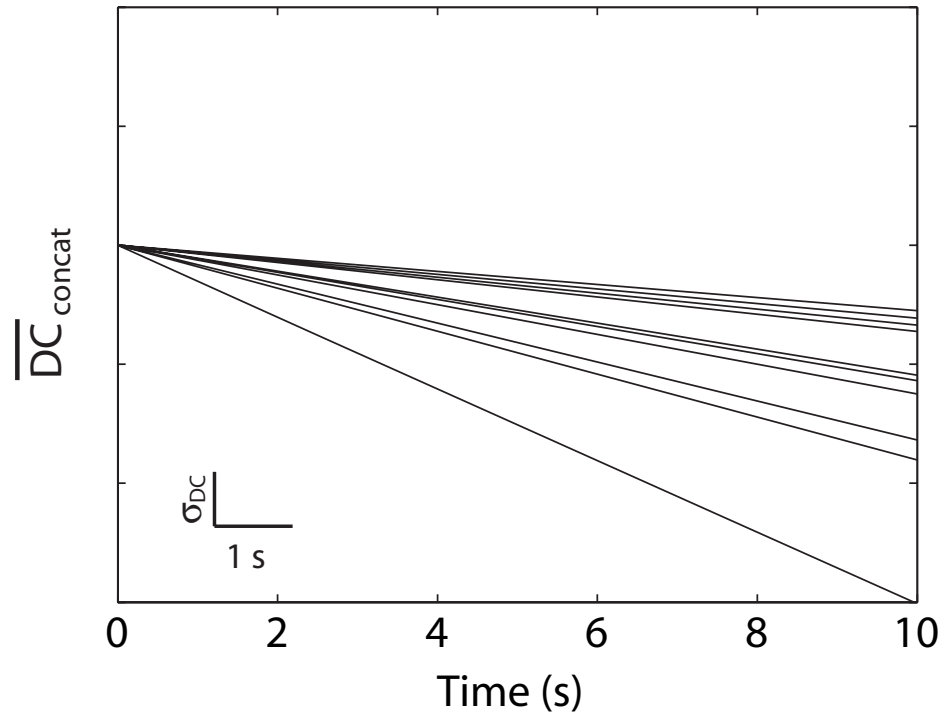

Fig. 8. Representative non-adapting time-courses for the $\overline{D C}$ of the response to both low and high modulation depth components computed from Eq. 4 with parameters $\mathbf{a}, \mathbf{c}$, and $\mathbf{d}$ drawn from their respective distributions. $\mathbf{c}$ and $\mathbf{d}$ were selected from their distributions corresponding to $|\mathbf{d}| \leq 0.006 \mathrm{~s}^{-1}$. 

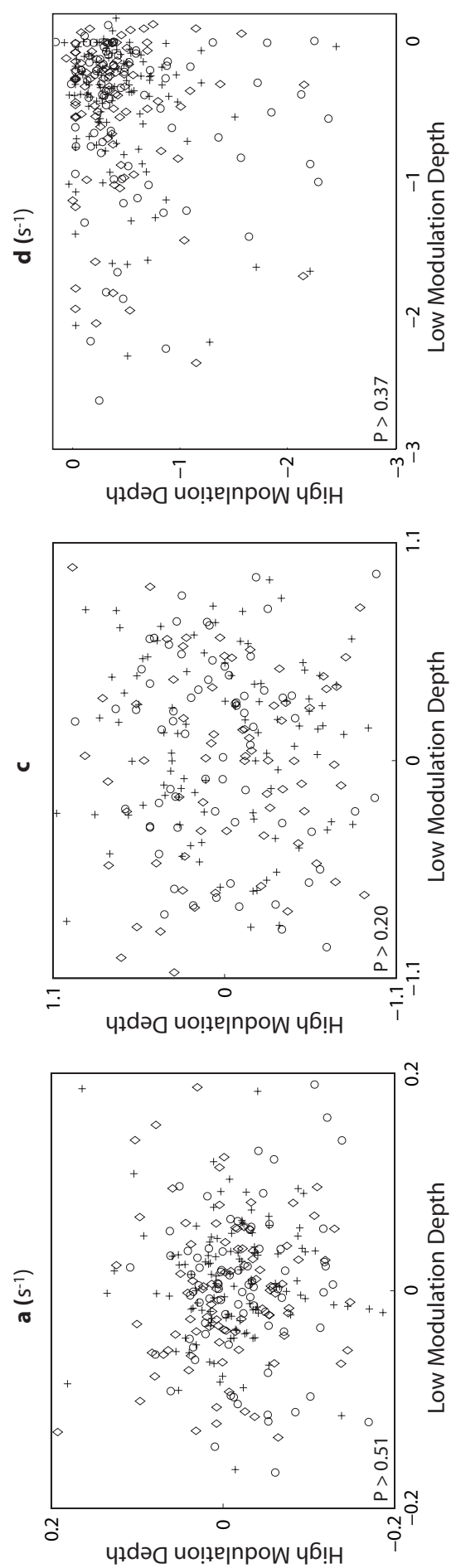

Fig. 9. Distributions of second-order adaptation parameter values for the $\overline{A C}_{1}$ of the response grouped by stimulus bandwidth. The parameters for the low modulation depth components of the stimulus are plotted against the parameters for the high modulation depth components. The distributions for all three parameteres were not significantly different at different bandwidths of the stimulus (MANOVA, $P>0.51$ $[\mathbf{a}], P>0.20[\mathbf{c}]$, and $P>0.37[\mathbf{d}]) . B W=0(+), 0<B W \leq 2(\circ), B W>2(\diamond)$. Bandwidth in octaves. 

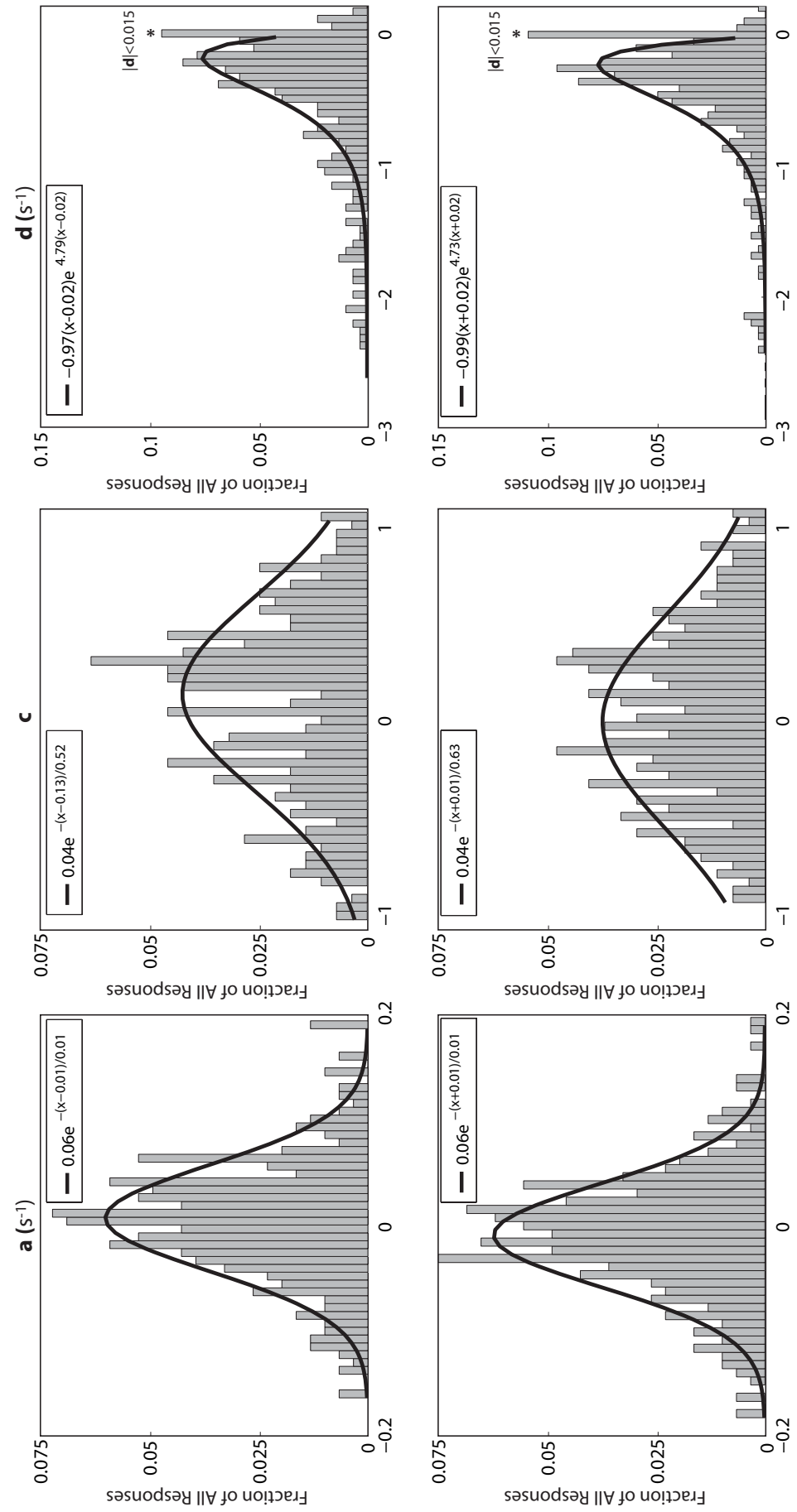

uzdəa uo!̣ejnpow MOך

чұdəa uo!̣e|npow ч6!!

Fig. 10. Distributions of second-order adaptation parameter values for the $A C_{1}$ of the response to stimuli with low and high modulation depths. For low modulation depths, the mean of a was $0 \pm 0.06 \mathrm{~s}^{-1}$, whereas for high modulation depths, it was $-0.01 \pm 0.06 \mathrm{~s}^{-1}$. There were two populations observed in $\mathbf{c}$ and $\mathbf{d}$-nonadapting (for which $|\mathbf{d}|<0.015$ ) and adapting (for which $|\mathbf{d}|>0.015$ ). In the adapting responses, c had a mean of $0.05 \pm 0.48$ and $0.03 \pm 0.45$ for low and high modulation depths, respectively. There was a slight positive shift in $\mathbf{d}$ values upon transition from low to high modulation depths, but the shape of the distribution overall remained unchanged. 


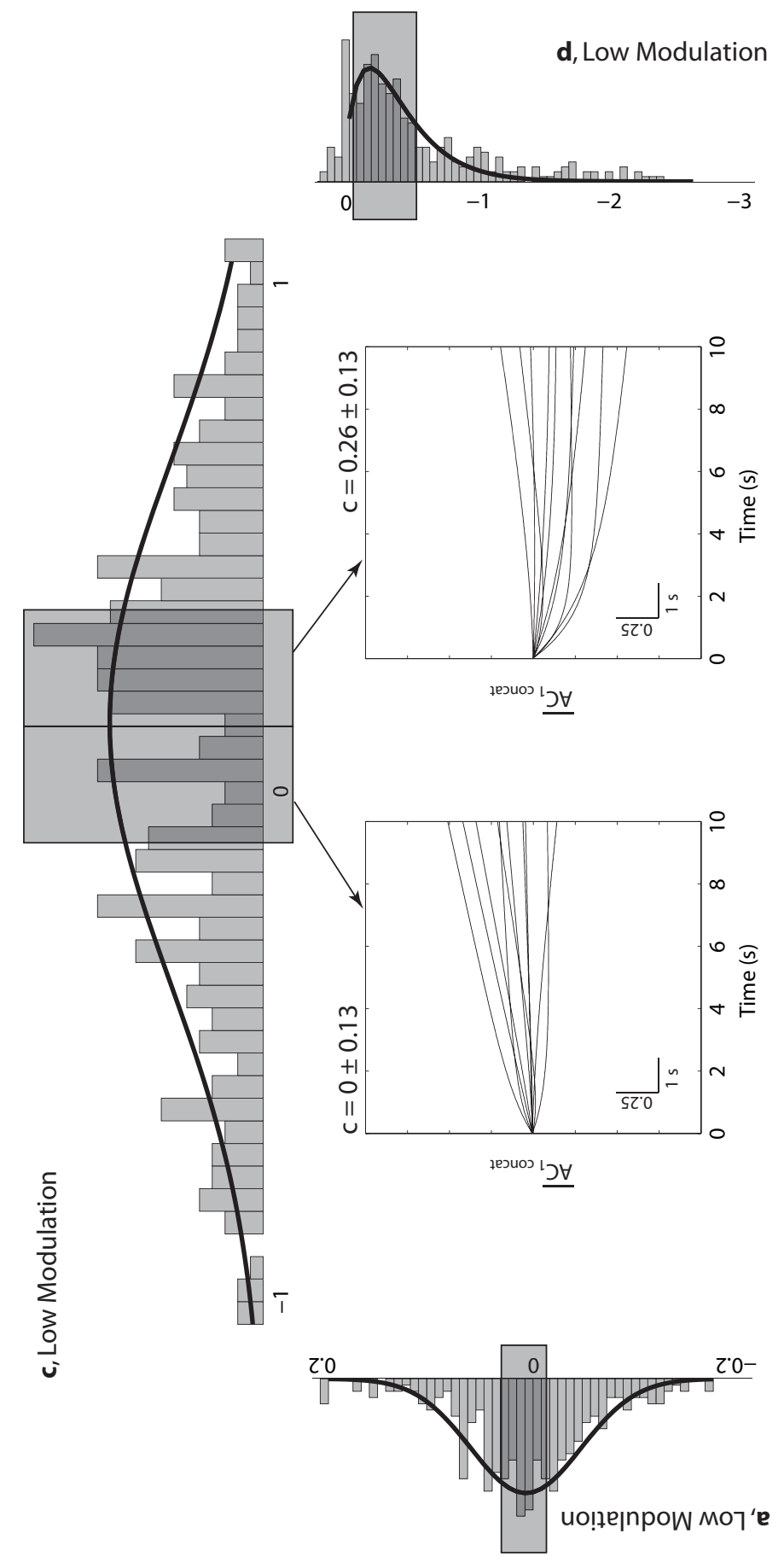

Fig. 11. Representative time-courses of adaptation for the $\overline{A C}_{1}$ of the response to low modulation depth components computed from Eq. 4 with parameters a, c, and d, drawn from their respective distributions. In contrast to the $D C$ of the response, the distribution in $\mathbf{c}$ was unimodal. Sample groups for each parameter were taken at the mean $\pm \sigma / 2$. 

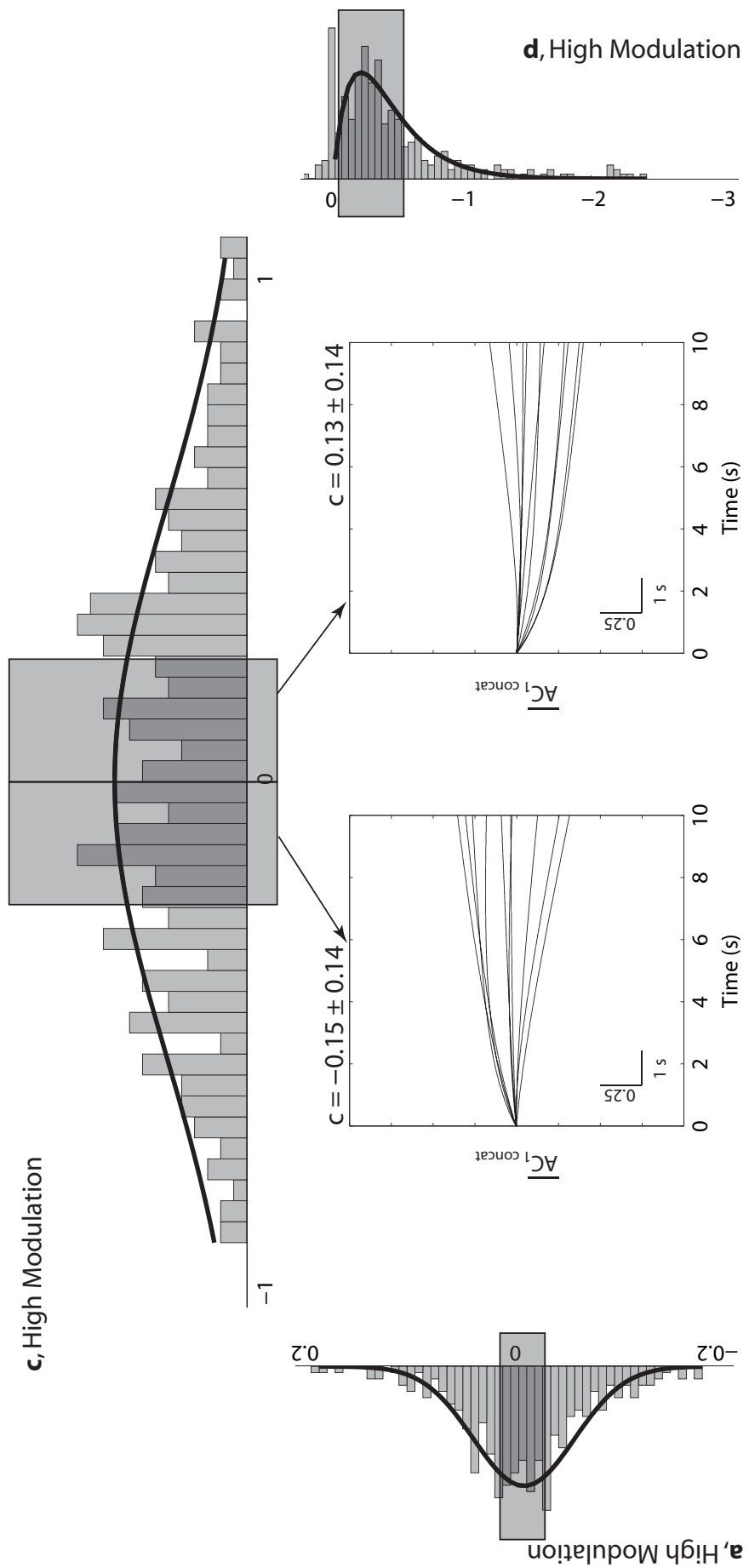

Fig. 12. Representative time-courses of adaptation for the $\overline{A C_{1}}$ of the response to high modulation depth components computed from Eq. 4 with parameters a, c, and $\mathbf{d}$, drawn from their respective distributions. Sample groups for each parameter were taken at the mean $\pm \sigma / 2$. 

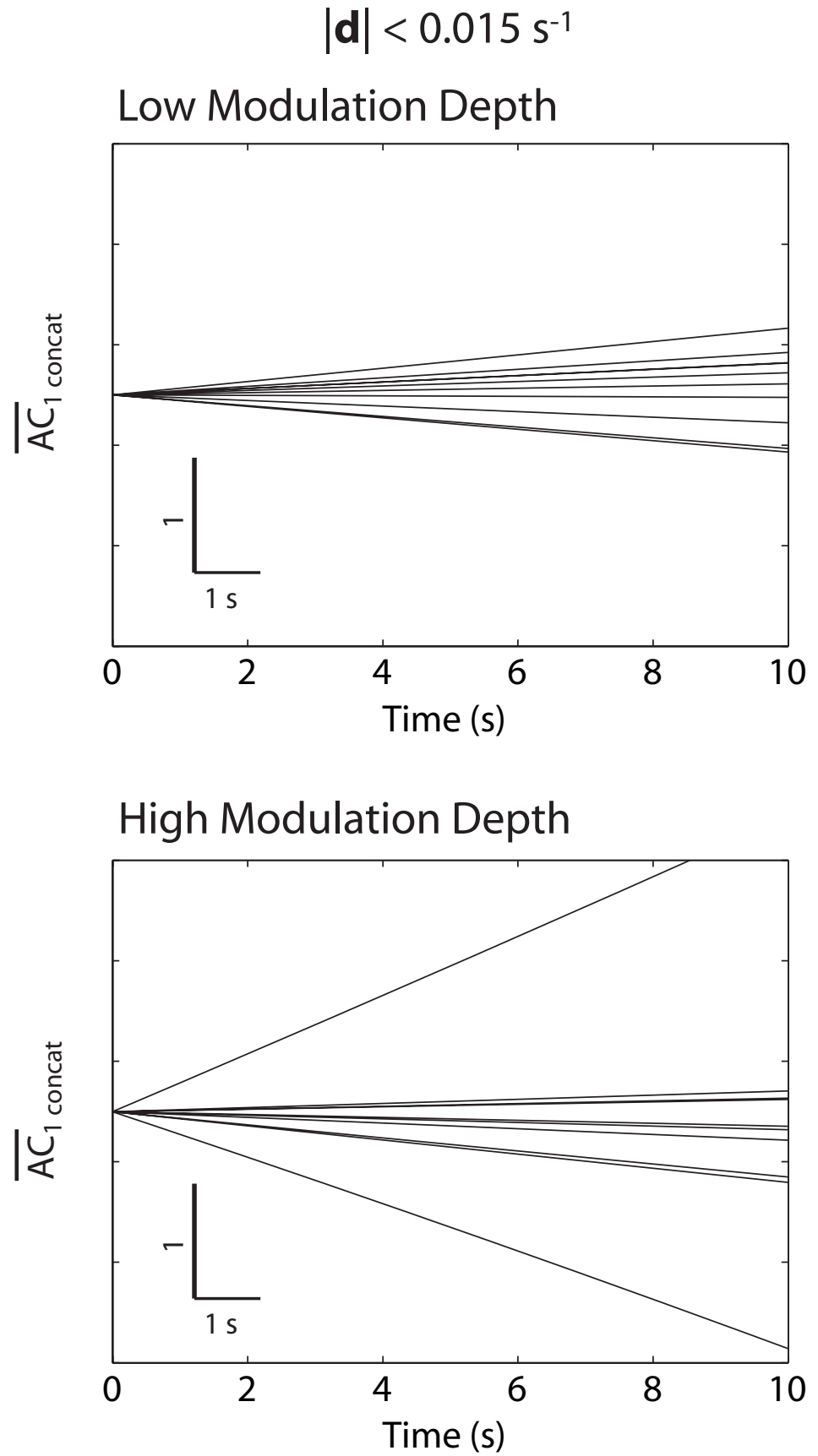

Fig. 13. Representative non-adapting time-courses for the $\overline{A C}_{1}$ of the response to both low and high modulation depth components computed from Eq. 4 with parameters $\mathbf{a}, \mathbf{c}$, and $\mathbf{d}$, drawn from their respective distributions. $\mathbf{c}$ and $\mathbf{d}$ were selected from their distributions corresponding to $|\mathbf{d}| \leq 0.015 \mathrm{~s}^{-1}$. 